LIVED EXPERIENCE

\section{Living with Polycystic Kidney Disease}

I t wasn't till my late 20s that I discovered what happened to my mom. While I knew she died from kidney disease complications, I did not realize she was misdiagnosed and one of her kidneys was removed. The induced stress on the remaining kidney sped the path to kidney failure. The disease now had a name "polycystic kidney disease (PKD)."

An ultrasound revealed that, indeed, I had multiple cysts in my kidneys. Quick research showed me that there was no cure. As a self-confessed control freak, this was difficult. I like to have an action plan. Here I was confronted with a disease that required me to simply wait-a virtual ticking time bomb! Apart from regular checkups with my nephrologist and brain aneurysm scans, as berry aneurysms have a well-known association with autosomal dominant PKD, I went about my life. Other than a niggling pain in my lower right abdomen and the fact that sleeping on my right side was uncomfortable, I appeared healthy. I was a busy woman; I had kids to raise, field trips and soccer practice, and volunteer art lessons to give, all the while working full time. I had no time for an invisible disease, although I made sure I never missed any health appointments.

During a regular checkup in my late 30s, my blood pressure was high. It was time to start monitoring it regularly, and perhaps the first sign of more to come. I was put on medication to control my blood pressure, but due to a low resting heart rate and PKD, I had a small range of medication options. My low heart rate necessitated a visit to the cardiologist; echocardiograms and stress tests revealed no problems. I simply had the resting heart rate of a professional athlete. Regular visits to my nephrologist became part of my routine. My kidney tests remained mostly normal, though I would increasingly need more medications to control my blood pressure.

As I approached my mid-40s, things progressed slowly. With no cure in sight, a reassessment of my current strategy was needed. A conscious decision to dedicate myself to a healthier lifestyle led to regular exercise. Soon, I was jogging 4 days a week while training for marathons and spinning twice a week. To defeat my invisible enemy, fitness was my weapon. By this time, my kids were in college, and I had more time for myself.

As I entered my 50s, my PKD was advancing. Was it going to be quick or slow? No one knew, as that's the nature of the disease. I had surpassed my mother by 11 years, my kids were college graduates, my son had completed his Masters, and I had run five marathons. I wasn't doing too badly.

In the fall of 2017, my estimated globular filtration rate (eGFR), a measure of kidney function, hit below 20 for the first time, making me eligible for the kidney transplant list. I chose the nearby Cedars-Sinai Comprehensive Transplant Center, Los Angeles, CA, USA. Kidneys are precious commodities, so the evaluation, education, and testing processes are rigorous. By March 2018, I was listed for the kidney transplant. With a wait time of $8-10$ years to receive a deceased donor kidney, my other option was to find a living donor. My family set out to create a campaign to find me a donor. A flurry of emails and a website were created to generate awareness of my need for a kidney.

With my eGFR in a nosedive, my nephrologist recommended preemptive access creation for dialysis. Two surgeries later, in the summer of 2018, I had an arteriovenous (AV) fistula - the gold standard of access - and the possibility of dialysis looming. I wanted to put off dialysis as long as possible, and my nephrologist reluctantly agreed I could wait till the New Year.

On January 2, 2019, my access was inducted as my lifeline at my first dialysis session. My new routine was now this: on Mondays, Wednesdays, and Fridays, leave for work at 7:30 am, end my workday at 5:00 pm, make a mad dash through rush hour traffic for a $4 \mathrm{hr}$ dialysis session, and end my day around 10:00 pm. It took all I had to muster enough energy for lunchtime walks and 1-2 spin classes per week. I was drained, but wasn't ready to throw in the towel yet. In addition to the low-protein diet to reduce stress on my kidney, I limited my intake of potassium, phosphorus, and sodium.

Luckily, I received news that I had a living donor soon after starting dialysis. However, my PKD had progressed significantly since I got listed. PKD causes an overgrowth of cysts on the kidneys, enlarging them as much as an American football. I looked 6 months pregnant due to my oversized kidneys. My surgeon recommended a double nephrectomy, with a 2- to 4-month recovery, prior to transplantation.

Dialysis after the double nephrectomy was very different. All fluids ingested, as well as impurities and waste in the blood, are removed by dialysis. Excess fluid removal can lead to cramps and other adverse reactions. A strict water intake restriction was instituted to help control my fluid intake. Still, I was alive and thriving. 
As I acclimatized to dialysis, I was ready to resume travel abroad that dialysis had put a damper on. Advance planning to arrange dialysis was necessary, but I was eager to make it work. Over the next 3 months, I planned three trips: Calgary in June, Ghana in July, and St. Lucia in August, a whirlwind of travel!

As my transplant surgery approached, I scheduled my preoperative visit just before my last trip, the perfect ending to months of dialysis. The preoperative visit went well, but 2 days into my vacation, I received a devastating call from the hospital, informing me that the transplant surgery was cancelled. I had developed strong antibodies to my donor from a blood transfusion I received after my nephrectomy. Crushed is probably the best word to describe my feelings.

Ensuing conversations with the transplant team revealed two options: find a new donor or enter the paired kidney exchange (PKE) program where recipients with incompatible donors swapped kidneys. My family started a new campaign to find a donor, and I entered the PKE program simultaneously. A prerequisite of the program was a 6-week treatment course of rituximab and intravenous immunoglobulin (IVIG) to rid my body of antibodies. I was emotionally exhausted by the start of the holiday season, and the antibody treatment left me feeling weak and unwell. By the New Year, I was beginning to feel better as the treatment effects wore off. A few days later, I had a match in the PKE program, involving a total of eight recipients and donors. I was cautiously optimistic.

In January 2020, I finally had my kidney transplant! I am one year post-transplant and doing well. The medications I need have dropped significantly. On any given day,
I feel better than my pre-transplant days. I am exercising again and looking forward to reaching my previous fitness level. I am back to working full-time, remotely as a result of COVID-19. In my free time, I have been working with my family to create a resource cataloging my journey with PKD to serve as an inspiration to people on their own PKD journeys.

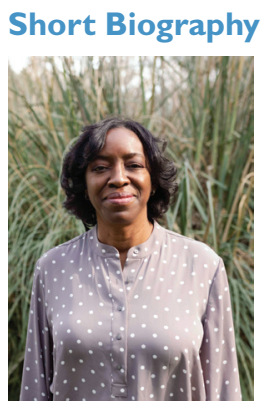

Olufunke Ojuri is a Civil Engineer based in Los Angeles, CA, USA, where she currently serves as the Chief of Civil Design with the U.S. Army Corps of Engineers working on flood risk management projects. She received a B.S. in Civil Engineering from Obafemi Awolowo University, Ile-Ife, Nigeria, and received an M.S. in Civil (Structural) Engineering from Stanford University, Stanford, CA, USA. Outside of work, she is working on an initiative to provide resources for patients struggling with PKD. Olufunke had a double nephrectomy in April 2019 followed by a kidney transplant in January 2020 and is currently on the road to recovery. During her free time, Olufunke enjoys traveling abroad, running marathons, cooking, and spending time with loved ones. Olufunke is married and has two children.

\section{Olufunke Ojuri}

fojuri@yahoo.com 